\title{
VERGINE GIURATA DE ELVIRA DONES: EL TERCER SEXO EN ALBANIA
}

\author{
VERGINE GIURATA BY ELVIRA DONES: THE THIRD SEX IN ALBANY
}

Yolanda Romano

Universidad de Granada

\section{Resumen:}

Elvira Dones es una escritora y guionista albanesa. Nacida en el seno de una familia privilegiada en pleno apogeo de la Albania comunista, Elvira viajó por distintos países hasta establecerse en una región de Suiza de habla italiana. Entre sus obras destaca Vergine giurata, en la que describe las dificultades de la condición femenina en su país natal.

\section{Palabras claves:}

Elvira Dones, Albania, mujer.

\section{Abstract:}

Elvira Dones is an Albanian writer and scriptwriter. Born in a privileged family at the peak of the communist Albany, Elvira travelled by different counties till she settled down in an Italian-speaking region in Switzerland. Among her works we can underline Vergine giurata, in which the difficulties of woman condition in her home town are described.

\section{KEY WORD:}

Elvira Dones, Albany, woman. 


\section{Albania: el país europeo más desconocido}

Albania (en albanés Shqipëria) es una república del sudeste de Europa que limita con Montenegro al norte, Serbia (incluyendo Kosovo) al noreste, la República de Macedonia al este, y Grecia al sur. Está bañada por el mar Adriático al oeste, y el mar Jónico al suroeste y su capital y mayor ciudad es Tirana. Estos son los datos que nos sitúan en este pequeño país, cercano geográficamente pero muy lejano desde el punto de vista cultural. Su perfil geográfico está caracterizado por cadenas montañosas que atraviesan el país pero que a su vez hacen de frontera, de barreras físicas que favorecen la separación de los territorios vecinos. Sin duda un gran desconocido, un espacio aislado e indiferente a los ojos del resto del continente. Desde hace años lugar excluido del mapa por su historia política y sobre todo por su carácter de país primitivo de los Balcanes.

Hasta la caída del régimen comunista, Albania había venido a representar no sólo una frontera cerrada de Europa y del Mediterráneo sino también del resto del mundo, donde la libre movilidad de las personas estaba prohibida. Desde los años 80 el mayor flujo de inmigrantes que llega a Italia proviene del país del águila bicéfala. Entre estos hombres y mujeres que han abandonado su país en busca de mejor suerte se ha formado con el tiempo una generación de escritores que han elegido dar a conocer sus vivencias y sus raíces en la lengua de acogida: el italiano. Estos autores han logrado abrirse paso en el complicado terreno editorial presentando obras que narran la visión del exiliado, del migrante y a su vez han dado a conocer la cultura que se han visto forzados a abandonar.

\section{Elvira Dones: una escritora transnacional}

Perteneciente a la generación de autores migrantes la escritora y guionista Elvira Dones es la autora albanesa más conocida en Occidente. Nace en la ciudad marinera de Durrës en 1960 y crece en Tirana en el seno de una familia privilegiada ligada al régimen y al partido comunista. Su padre era un ingeniero químico con un importante trabajo para el régimen incluso desde la perspectiva militar. Obsesionada por la lectura, comienza a leer muy pronto olvidando los juegos de niños y prefiriendo observarles. A los 10 años inicia sus estudios de italiano y con esta edad escribe su primer 'libro' de una docena de páginas que titula 'Romanzo', una historia comunista, la de un niño pobre rescatado por el socialismo. Crece por tanto como hija del régimen entre los privilegios que el partido le otorgaba a su familia. Como ella misma ha confesado hasta los 17 años era una 'bambina indottrinata' a la que su padre advertía por hablar demasiado. Diferentes sucesos en su vida de estudiante le permiten a abrir los ojos y darse cuenta de la realidad en la que vive.
Su contacto con el mundo audiovisual le llega presentando programas en la televisión albanesa que compagina con sus estudios universitarios. Se gradúa en Letras Albanesas e Inglesas en la Universidad de Tirana y durante su etapa universitaria, el país estaba controlado por el dictador comunista Enver Hoxha, que lo gobierna durante más de cuarenta años, hasta su muerte en 1985. Emigra de su país en 1988 antes de la caída del Muro de Berlín cuando aún pervive una dictadura estalinista. Dones entonces periodista en el canal de televisión del estado huye, en parte por frustración con el gobierno albanés por la falta de libertad que sufren los medios de comunicación:

Lasciai l'Albania nell'ottobre del 1988, era ancora una dittatura, io avevo 28 anni. Non sapevo assolutamento cosa mi aspettasse dall'altra parte del muro. Non mi ponevo nemmeno la domanda, ero troppo impegnata di sogni e di ingeniutà perchè cresciuta con il sogno del mondo con le parole dell'italiano dentro, con le parole dell'inglese, del francese e dello spagnolo che cercavo di studiare a grande fatica perché ci mancava ciò che fa vivere una lingua: la comunicazione diretande

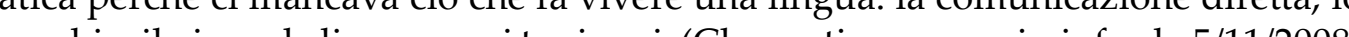

Se traslada a una región Suiza de habla italiana donde vive y trabaja para la televisión hasta el año 2004. Durante su permanencia allí escribe siete novelas, dos libros de relatos y diversos guiones así como documentales para la televisión suiza. Su paso por Suiza le supone también afianzar sus conocimientos del italiano y su metamorfosis como autora que escribe en su lengua madre albanesa Estos 16 años trascurridos en este país le han dejado huella, hasta el punto de sentirse como en casa, porque aprecia su precisión y disciplina suiza.

\section{Ho imparato a utilizzare la svizzerità per temperare la mia emotività balcanica. Per uno scrittore è infatti fondamentale trovare l'equilibrio e situarsi alla giusta distanza dalla storia che desidera raccontare: io tornavo al mio paese per fare scorta di storie dalla viscerale drammaticità, e poi rientravo in Svizzera per scriverle.} Clementi, www.swissinfo.ch, 5/11/2008)

Está casada y es madre de dos hijos, con los que vive en la actualidad en Washington D.C.. Divide su tiempo entre los Estados Unidos, Suiza, Italia y Albania y alterna la narración con su trabajo de periodista, guionista y documentalista. Con esta última actividad ha logrado numerosos reconocimientos internacionales por diversos documentales televisivos, algunos de ellos ligados a la cultura y a la historia reciente albanesa. Para la televisión suiza realiza junto al director de cine Mohamed Soudani 'Cercando Brunilda' (2003) donde aborda el tema del tráfico de prostitutas entre Albania e Italia en los últimos años. Con él logra ser finalista en el Premio de Periodismo Ilaria Alpi en 2004. Producido y difundido por la RSI radiotelevisión suiza de habla italiana realiza en colaboración con el director de cine Fulvio Mariani el documental 'I ngujuar' ('Inchiodato') (2004) sobre la venganza de sangre en la Albania del Norte, con el que 
obtiene diversos galardones: el premio Fipa d'argent en el Festival De Programas Audiovisuales de Biarritz y el premio Reportage del Mediterráneo de Siracusa ambos en 2005. Ganador del premio como mejor documental en el Baltimore Women's Film Festival del 2007 es su trabajo titulado 'Vergini Giurate' (2006) coproducido por la RSI y Dones Media.

Tras escribir ocho libros de ficción ha consolidado su carrera literaria alcanzando una gran popularidad en su país y en Italia. Indudablemente una de las características que han marcado profundamente su personalidad y su actividad profesional es el exilio al que se va a aferrar como tabla de salvación al igual que han hecho durante años otros miles de albaneses:

\section{Diciannove anni fa, quando lascia la mia terra, io diedi un taglio netto. Fu un dolore atroce, ma allora non c'era un'altra opzione oltre la fuga. (Lepori, http://} www.culturactif.ch, 4/01/08)

Junto a otras autoras albanesas como Anilda Ibrahimi, Leda Apostoli, Anila Anxari, Lindita Mamli o la premiada Ornela Vorpsi ha dado a conocer en sus novelas la reciente y compleja historia albanesa eligiendo para ello la lengua de Dante. El caso de Elvira Dones es, en cierto modo diferente, dado que a pesar de ser una autora migrante vive en el exilio por decisión propia y la mayor parte de su producción está en lengua albanesa. Muchas veces se le ha interpelado sobre su posible pertenencia a la categoría de autores migrantes pero ella rechaza las etiquetas:

\section{Le categorizzazioni sono dei modi per ghettizzarti. Io direi di far parte nella schiera degli scrittori transnazionali, internazionali, o anche scrittori e basta. Sono degli scrittori che portano dentro un po' del mondo da cui provengono, e un po' di quello in cui sono arrivati. Abbiamo bisogno di queste storie multi-colori. (Rukaj,} www.osservatoriobalcani.org, 13/11/08)

No obstante, Dones considera proficuo para la cultura de un país la presencia de autores extranjeros que escriban en italiano puesto que muchos de ellos han devuelto la vitalidad y la frescura a la literatura italiana. Los escritores migrantes han contado lo que dejaron atrás pero también su nueva vida con sus luces y sombras. Se han despojado de sus lenguas maternas el albanés, el serbo-croata o el rumano quedando relegadas a lenguas de la infancia, de la memoria y de un pasado doloroso. En sus albores las historias que estos autores trasmiten está ligadas al su vida anterior pero hoy la mayor parte cuenta sus vivencias actuales en el país que les acoge. Esto sin duda enriquece la historia literaria de una nación asumiendo sabores y sonidos nuevos.

En Italia ha publicado diversos libros, traducidos del albanés (de los que ella misma se ocupa de la traducción). Su ópera prima es Senza bagagli de 1998 (Besa) cuyo original publica un año antes con el título Dashuri e huaj. Definido por los críticos 'romanzo- confessione' narra la historia autobiográfica de una operadora cultural albanesa, Klea Borova, que en los años 80 en pleno comunismo vive en su país con todo lo que una mujer podría desear. Un día recibe el regalo más preciado que su partido podía conceder a una mujer: el derecho a viajar al extranjero para asistir a un congreso. Será en su estancia en Dinamarca donde descubra el amor y traicione a su República, a su familia, a sus amigos y a su propio hijo. Estos últimos pagarán las consecuencias de su insano gesto. Nunca más regresará a Albania. El Catón Ticino en Suiza se convertirá en su hogar y será el inicio de una nueva vida en libertad. Lejos de su amada tierra vivirá para escribir.

Su segunda novela Sole bruciato de 2001 (Feltrinelli) nada tiene que ver con la anterior puesto que el odio, la violencia y la maldad son sus principales ingredientes. Nos presenta los sueños rotos de las jóvenes prostitutas albanesas que hacen la calle en las aceras de Europa. Cuenta la historia de Leila, una joven albanesa que es engañada y obligada a prostituirse en Italia y que tras tres años regresa en un ataúd a su país. Con un lenguaje épico y brutal, onírico y cruel nos obliga a hacer un examen de conciencia sobre una situación vergonzante en la sociedad occidental.

La trama de su tercera novela, Bianco giorno offeso (Interlinea) en 2004, gira en torno a la profunda y tormentosa amistad entre Illir Bejko un prófugo albanés y Max Baumann un joven perteneciente a la burguesía de Lugano. Pone en escena la amistad sin límites, los amores imposibles, el dolor y la destrucción con lo que consigue tocar el corazón del lector. En sus páginas la autora nos revela el drama de la fuga y de vivir como un refugiado en un país extranjero, que son en definitiva sus propias vivencias. Este libro representa la segunda parte de su Trilogía del dolor iniciada con Sole bruciato con la que realiza un labor de denuncia, un duro llamamiento a la sociedad para que tome conciencia de la realidad de sufren algunas mujeres. La tercera parte $E$ dopo il silenzio sólo se ha publicado en Albania.

En I mari ovunque de 2007 (Interlinea) un viaje a Irlanda de sus dos protagonistas, Eric y Andrea nos sirve de trasfondo para entender la vida y las elecciones a través de continuos flashbacks. Andrea es una joven huérfana argentina que ha crecido entre el dolor y el sufrimiento, con Eric encontrará la normalidad y la estabilidad desconocida para ella.

Ma con un simile mattino, lì, sotto il cielo di Dublino, Andrea si sentì disarmata. Una mano onnipotente le scagliava nell'animo ogni sorta di meraviglia, sabotando i suoi buoni propositi, e lei non voleva essere ostacolata. Bastava la presenza di i suoi buoni propositi, e lei non voleva essere ostacolata. Bastava la prese
Eric, pensò, a incarnare tutti gli ostacoli di questo mondo. (Dones, 2007:12)

Una historia triste pero al mismo tiempo emocionante contada con gran sensibilidad y que nada tiene que ver esta vez con su Albania natal. La gestación de sus libros nace 
de las personales experiencias de la autora, de su sufrimiento, de sus vivencias pero no se conforma con contar su historia sino que pretende contar la del mundo que le rodea, sin perder nunca su punto de referencia natal:

Io sono una viaggiatrice nata, nella vita e in ciò che scrivo. Mi è capitato di occuparmi dei miei dolori, come spesso fanno gli scrittori. Ma per relativizzare i miei, mi sono occupata anche di quelli degli altri. Gli spunti sono numerosissimi. In particolar modo noi scrittori dei Balcani siamo molto fortunati perché proveniamo da una terra ricca di storie e di culture. (Rukaj, www.balacanicaucaso.org, 13/11/08)

\section{Vergine Giurata: vivir como un hombre}

Su primera y única novela escrita directamente en lengua italiana publicada en 2007 se titula Vergine giurata (Feltrinelli). En ella aborda la temática de las dificultades de la condición femenina en Albania, cuyo caso más extremo son las vírgenes juradas. Las 'burrneshë' son mujeres de la Albania más profunda de las montañas obligadas por tradición o por necesidad a travestirse y vivir como hombres para sacar adelante a la familia. Para su libro, Dones investigó esta desconcertante tradición, que ha sido documentada por historiadores y sociólogos, adentrándose así en el territorio poco conocido para el resto de los europeos de la cultura balcánica. El libro es un viaje al interior de la Albania más profunda, hacia un pequeño pueblo ubicado en las Montañas Malditas llamado Rrnajë, donde el tiempo parece haberse parado y donde la única ley que se respeta es la del Kanun. Este código medieval que pervive en algunas regiones del norte del país y en Kosovo desde el siglo XV es un conjunto de leyes que se ha trasmitido de generación en generación pero no se codifica ni se transcribe hasta el siglo XIX. Este código se divide en secciones: Iglesia, Familia, Matrimonio, Casa, Ganado y Propiedades, Trabajo, Transmisión de la Propiedad, Palabra, Honor, Daños, Ley concerniente al Crimen, Ley Judicial y Exenciones y Excepciones. Las normas del Kanun, alejadas de cualquier forma de religión, ya sea católica o musulmana, son las que han regido durante toda la historia moderna del país. Subsisten aún hoy en las zonas más remotas en territorios de pastores y montañeses, por la pérdida de la confianza en los gobiernos locales. El Kanun defiende un sistema patriarcal, regido por hombres donde el rol de la mujer es de mera sierva, de sumisión total a la voluntad del hombre. En este pueblo mísero de las montañas malditas regido por las leyes estrictas del Kanun se desarrolla una parte de la historia de la joven Hana donde vive con sus tíos Gjergj y Katrina Doda en una kulla tradicional, una casa humilde. Sus tíos son los que la han criado desde que sus padres murieran en un accidente de coche no obstante mantienen una relación con Hana de afecto filial. Hana adora a sus tíos y ellos quieren un futuro diferente para ella por ello acceden a que estudie en la Universidad de Tirana.
Gjergj Doda y Katrina Doda viven del campo en unas condiciones muy humildes y respetando las costumbres más austeras propias de los montañeses. En la casa de los Doda no hay libros, sólo una Biblia bien escondida y una historia del héroe nacional Skanderberj.

En diversos pasajes del libro conocemos su relación con el régimen político de su país. Intuimos que Gjergj Doda no es muy partidario del régimen a pesar de no haberse pronunciado jamás en contra. Su fe católica le convierte en sospechoso de ello dado que los católicos son mal vistos por el partido.

Era un buon contadino: si atteneva alle regole dei comunista, con l'eccezione del nome affibbiato al capretto, che di nascosto aveva chiamato Enver. Ma questo era un dettaglio che nessuno conosceva. (Dones, 2007:80)

El drama de la joven Hana comienza en 1986 cuando regresa de forma precipitada de Tirana, donde estudia letras en la Universidad, para descubrir que su tío está muy grave; padece un cáncer de garganta:

Ora Hana vede il collo gonfiato da far spavento, lo sforzo per muovere normalmente la mascella mentre parla. Lui vuol sapere dell'Università e lei gli responde che fra pochi giorni avrá un importante Seminario sul Rinascimento letterario albanese. (Dones, 2007:58)

Su enfermedad es irreversible pero Hana no se da por vencida y lucha para que su tío tenga alguna esperanza. Para ello cuenta con el apoyo del médico del pueblo un joven de 30 años que desterrado allí por los problemas de su familia con el régimen, añora la vida su anterior vida en Tirana. El tratamiento que debe seguir, obliga a Hana a esperar una costosa medicación que proviene una vez al mes de Shkodër (Scutari en italiano). Pero Hana no se rinde, una vez pasado el duro invierno, consigue llevarle al hospital civil de Tirana. El regreso a la capital le supone el reencuentro con su pasado, con aquella vida universitaria que había interrumpido bruscamente. Hana se topa con la intransigencia de la secretaria de la facultad donde estudia porque no le permiten tantas ausencias injustificadas. Ante esta situación Hana debe reivindicar su sentimiento de hija legítima de los Doda puesto que ellos son los que la han criado desde que fallecieran sus padres en un accidente, algo que no entienden ni en el Hospital, ni en la Universidad:

Il malato è l'uomo che mi ha cresciuta, quindi è mio padre. (Dones, 2007:66)

È mio padre. Ho detto che è mio padre. (Dones, 2007:68)

A pesar de la negativa inicial de Gjergj de dejarse operar para prolongar su vida, Hana consigue convencerle: 
Fatti operare per me, ti scongiuro. Sono quarant'anni che tieni una pallottola in corpo e non ti sei mai lamentato, cosa sarà mai un bisturí, per te? (Dones, 2007:69)

Descubrimos en estas páginas a una joven enamorada de su ciudad, que ama la literatura, que le gusta escribir poesía y como cualquier otra joven tiene grandes esperanzas de formarse y de tener un futuro mejor.

Qui puoi scoprire nuove passioni e fare nuove conoscenze, come Hikmet, come Neve, come le nuove parole della sua lingua, come gli scrittori che sui monti non verranno mai. (Dones, 2007:71)

Una espléndida mañana de primavera los Doda regresan en una ambulancia a su pequeño pueblo dejando a Hana en la capital. Aquella será la última vez que vea a su adorada tía Katrina puesto que muere de un infarto la tercera semana de junio. Es entonces cuando verdaderamente el mundo se le derrumba y su vida deberá dar un giro radical. Faltándole un examen para terminar su primer curso en la Universidad regresa a su pueblo precipitadamente pero no logra llegar al funeral de su tía. Tras el desconcierto lógico del duelo Hana reencuentra al médico del pueblo que no duda en declararle su amor antes de su traslado a otra ciudad. Pero la joven, a pesar de sentir afecto por él, le rechaza bruscamente cuando le confiesa que pretende pedir su mano.

Al dolor extremo por la pérdida de Katrina se le añade ahora el agravamiento de la enfermedad de su tío. Pero Hana es fuerte, debe reaccionar en seguida y tomar conciencia de su realidad y del futuro que se le presenta. La decisión de ir a Scutari en busca de las medicinas para aliviar el dolor de su tío contravienen las férreas tradiciones machistas.

Lo so, zio, non devo osare. Sono una donna e devo stare zitta. (Dones, 2007: 87)

No obstante Hana se rebela, no entiende de normas, no puede quedarse mirando cómo se apaga su vida y al final viaje para conseguir la medicación necesaria. Gjergj le insta a volver a la Universidad a Tirana y allí Hana se reencuentra con aquél pasado feliz y con Ben un estudiante de francés del que estaba empezando a enamorarse que le pide que se quede más tiempo:

Lei corre. Fra sette, otto ore sarà a casa, e sarà salva. È bello andarsene, c'è qualcosa di eroico nella fuga, ti perdi via ti sfumi diventi nuvola oppure uomo. Ci vuole fegato per andarsene. (Dones, 2007: 99)

Hana huye de Ben al que no volverá a ver y de su pasado en Tirana ahora imposible. Nada en la vida de la joven volverá a ser como antes cuando Gjergj le anuncia que deberá casarse antes de que él muera.

Se non ti fidanzo adesso che sono in vita, dopo resterai senza marito e invece ci vuole un uomo per mandare avanti la baracca. Forse ho trovato a chi darti in sposa. Dopodomani arriverà il sensale. ... Voglio vederti sistemata, ho deciso. Non posso lasciarti sola. (Dones, 2007:101)

Estupor, rabia, desconcierto, odio, ira bullen en el interior de Hana. No puede dar crédito a lo que oye, pensaba que su tío era diferente:

Resterai sola. Una donna non sposata non vale niente. La donna è fatta per servire l'uomo e dargli dei figli. Non osare! (Dones, 2007:102)

Sei solo una donna! (Dones, 2007:103)

Pocas horas después su tío ya se arrepiente de la inaceptable propuesta y le hace jurar que se cuidará mucho tras su desaparición:

Farai l'uomo di questa casa. (Dones, 2007:104)

La salud de Gjergj sigue inexorablemente deteriorándose pero Hana le cuida sin desaliento día y noche. Cada vez son más pobres porque todo lo que tienen lo emplean en comprar los medicamentos por ello Hana decide trabajar en una Cooperativa Agrícola limpiando los establos. A la vuelta del trabajo descubre que deberá casarse a final del año con un maestro de escuela de un pueblecito cercano a Kosovo. El intento de violación que sufre por parte del camionero que la lleva a la ciudad en busca de las medicinas para su tío es seguramente el desencadenante de su decisión más dramática de su vida. Su violenta reacción como defensa nos descubre una Hana diferente llena de odio pero que ha entendido que no tiene sentido seguir siendo mujer en un mundo de hombres. El terror que ha vivido no quiere que se repita nunca más.

Así el 6 de noviembre de 1986 después de la agresión se presenta ante su tío vestida como un hombre; ya ha decidido ser una virgen jurada: Mi chiamerò Mark. Sarò Mark Doda. (Dones, 2007:111) Su tío acepta orgulloso y le entrega como símbolo de la metamorfosis el fusil que ha pertenecido a 6 generaciones de hombres del clan Doda. Nunca le hubiera propuesto una solución tan dolorosa puesto que Gjergj ansiaba un futuro mejor para ella en Tirana pero las circunstancias le obligan a asumirlo. A partir de ahora Hana aprenderá a ser un hombre, a comportarse y a moverse como tal. También comenzará un diario que servirá al final del libro para explicar sus razones y 
sus sentimientos al hombre del que se enamorará y definitivamente le devolverá a su condición femenina.

\section{En el pueblo enseguida se corre la voz, las mujeres evitan su mirada, los hombres} le saludan y repiten:

Onore a te per ciò che hai fatto. (...) Gjergj bre burrë, adesso hai un maschio e l'onore della kulla non morirà. (Dones, 2007:113)

Su única prima Lila no entiende su decisión pero Hana le recrimina su servilismo hacia su marido, su suegro y su cuñado. Lila representa en estos momentos para su prima lo que nunca aceptaría ser: una mujer sin derechos, al servicio del hombre. En mayor de 1987 Gjergj muere y el honor de los Doda queda a salvo porque ahora Mark goza del respeto de hombres y mujeres. Pero a escondidas Hana llora en silencio su soledad. Retomamos cronológicamente la historia de Mark/Hana en 1996 cuando Lila su prima que ahora vive en Estados Unidos con su marido y su hija le insiste en diversas cartas para que abandone su vida de hombre y se vaya a vivir con ella y su familia. Tras 10 años como Mark empieza a dudar de su existencia y aquella drástica decisión.

La visita inesperada de Blerta, una antigua compañera de la Universidad que ha venido a realizar un reportaje sobre el Kanun, va a empezar a derrumbar su férrea voluntad de ser una virgen jurada. A pesar de un desencuentro inicial, Blerta le abre los ojos y Hana le abre su corazón. Hana se sincera por fin libremente y le confiesa sus años vividos como un infierno, pero no explica porqué quiso ser un virgen jurada. Sabe que el Kanun no le permite volver atrás: Quel che è fatto non si puó disfare. (Dones, 2007:158)

A partir de ahora Mark vivirá en un mar de dudas e indecisiones hasta que en octubre de 2001 lo encontramos en un vuelo directo a Washington. Durante ese trayecto conoce a un hombre, Patrick O'Oconnor que será el que cambiará de nuevo su vida. Hasta que Mark logra la metamorfosis total y vuelve a ser Hana deberá pasar un tiempo. Contará con el amor y el afecto de Lila, su esposo Shtjefën Dibra y su sobrina Jonida. Un primer paso será la paulatina transformación física, volver a ser aquella joven atractiva de su época universitaria en Tirana, para después afrontar la revelación de su verdadera identidad femenina a su sobrina Jonida:

Ho fatto giuramento di non sposarmi mai, è un'usanza che esiste solo nel Nord del paese. Ti spiego: quando in una famiglia non ci sono figli maschi, una delle femmine giura di comportarsi da uomo e restare uomo per il resto dei suoi giorni. Da quel momento assume tutte le funzioni e tutti i ruoli di un maschio. Così io sono diventato il figlio che lo zio non aveva. (Dones, 2007:53)

Para la joven Jonida (de mentalidad occidental) su relato le parece absurdo e inaceptable, por qué motivo debería una mujer dejar de serlo por querer hacer las mismas cosas que un hombre? ¿Por qué una mujer no puede hacer simplemente lo que le da la gana en la vida? Escucha incrédula cómo Mark/Hana le expone que en Albania sólo los hombres pueden ser libres, mandar, comprar una tierra, defenderse, atacar, matar o dejarse matar; tienen la libertad, el deber y la gloria. A la mujer le queda sólo la obediencia y la sumisión. Y esto en 1986 para Mark/Hana era difícil de aceptar. Nunca una virgen jurada en Albania ha roto su juramento pues le supondría la muerte por ello esta segunda metamorfosis de Mark/Hana es tan difícil y dolorosa. Sobrecogida Jonida toma conciencia de una realidad absurda para una mentalidad occidental avanzada: hay mujeres en Albania que no tienen los mismos derechos que los hombres. A pesar de la ayuda que le presta su familia, Hana debe encontrarse a sí misma, recuperar la normalidad, reconocer su sexualidad. Ahora que su cuñado le ha conseguido un coche y un trabajo, a pesar de la desaprobación de su prima Lila, decide marcharse a vivir a un pequeño apartamento porque necesita estar sola. Su amor por los libros y su denodado esfuerzo por mejor su inglés le ayuda a obtener un trabajo mejor como dependienta en una gran librería.

Una última cuestión que le queda por resolver es la necesidad de tener una relación sentimental y sexual con un hombre. Para ello se pone en contacto con Patrick O'Connor aquél periodista con el que compartió su viaje a los Estados Unidos. La impresión positiva al verla cómo mujer presagia el comienzo de una relación no sin grandes dificultades. El diario que Hana escribe durante sus años como Mark sirve para que Patrick entienda su historia pero a pesar de todo, sus encuentros no resultan fáciles, por la inseguridad y la fragilidad emocional de Hana. En último capítulo del libro de Dones Hana salda las cuentas también en este sentido con su pasado y ahora sí está preparada para iniciar una vida nueva donde el amor verdadero estará presente. Dones pone punto y final a esta conmovedora historia de gran potencial dramático concediéndole a Hana un príncipe azul que la rescata y le devuelve su feminidad. La historia de esta Vergine Giurata concluye con una bellísima frase que resume lo que su corazón y su cuerpo de mujer siente:

È bello sapere di non dover morire. (Dones, 2007:204)

El retrato psicológico que Dones hace de Hana nos ayuda a entender este fenómeno desconocido para los occidentales europeos. Deja claro que esta forma de tercer sexo, como se ha definido, no tiene nada que ver con el travestismo o con la homosexualidad. Debemos interpretarlo como una renuncia a cualquier tipo de sexualidad. Hana en sus años de virgen jurada era una mujer vestida de hombre y con actitudes y ademanes de hombre porque le servía para poder sobrevivir en esa sociedad retrógrada. Si hubiera 
seguido siendo Hana no habría podido estar sola; se habría visto obligada a casarse con alguien que no amaba. El intento de violación que sufre es el espaldarazo que necesitaba para no querer seguir viviendo como aquellas mujeres sumisas sin voz y sin opinión, sin voluntad y sin futuro. Nos lleva a pensar que su elección no deja de ser una forma de rebeldía contra la voluntad de los hombres. Hana se pone la máscara de hombre simplemente para sobrevivir. Se niega a ser una mujer sometida como las demás, se niega a sufrir ningún tipo de violencia.

Sabemos que los catorce años como Mark los vive como un hombre verdadero y con los privilegios propios de su género: portar armas, trabajar, beber, fumar. Una virgen jurada renuncia a su sexualidad para obtener y gozar de grandes privilegios. Es respetada por hombres y mujeres. Pero quedan sin sernos revelados cómo es el mundo interior de esta joven virgen jurada: sus sentimientos, su soledad. Resulta muy interesante la forma en que Dones nos presenta la historia en continuos flashbacks puesto que al partir de un presente se nos va desvelando poco a poco su identidad; así mismo nos presenta en planos paralelos la doble metamorfosis: de Mark a Hana, de Hana a Mark. Recorremos el camino que Mark ha comenzado a emprender para volver a ser Hana y al mismo tiempo reelaboramos los obstáculos y las decisiones de su pasado como Virgen Jurada. El instinto de fuga que Mark/Hana demuestra al huir a Estados Unidos será la única forma para no sucumbir al peso de unas tradicionales ancestrales que perviven en el tercer milenio.

\section{Las Vírgenes Juradas en el tercer milenio}

La idea de escribir una historia basada en la leyenda de las vírgenes juradas se remonta a su época universitaria. En 1985 cuando aún reside en su país Elvira Dones intenta viajar al norte, a las montañas malditas, para contar la historia de las vírgenes juradas pero el régimen vigente que controlaba fuertemente a la prensa se lo prohíbe. Años después tras escribir este impactante libro y obsesionada aún por el tema siente la necesidad de conocer, de ver de cerca una virgen jurada. De su viaje de tres meses a la zona realiza un interesantísimo documental donde se constata que existen aún unas cuarenta vírgenes juradas, quizás alguna más en la zona de Kosovo, Serbia y Montenegro. En esta zona las condiciones de vida de las mujeres distan mucho de ser las que gozan las mujeres en el resto de Europa. Aquí las mujeres no han gozado de ningún derecho que les iguale al hombre. Son consideradas 'un saco hecho para aguantar': no pueden comprar tierras, no pueden votar, no tienen acceso a muchos oficios, ni siquiera entrar en muchos establecimientos. La discriminación de la mujer comienza en el propio ámbito familiar donde está siempre bajo la tutela de un hombre ya sea su padre, su hermano o su marido. Sufren la violencia y el maltrato continuo en su propia casa y ni siquiera tienen derecho a elegir marido. Dones quería conocer de primera mano es historia de estas 'otras mujeres', las burrneshë (de burrë, hombre) y para ello logra entrevistarse con una docena de ellas: desde ancianas a jóvenes veinte añeras. Dones nos revela que el fenómeno de las vírgenes juradas o ‘burrneshë' surgen por motivos diversos. A veces las niñas desde muy pequeñas eligen este rol masculino por tradición. En otros casos se ven obligadas por circunstancias familiares como por ejemplo el hecho de no existir otro varón en la casa o la muerte del patriarca. Este es el caso de Diana Rakipi, la sexta de nueve hijos, que tras la muerte de uno de sus hermanos pequeños decide prestar juramento en su recuerdo para ser un hombre tan bello como él. También algunas veces, las jóvenes (Virgjina = vírgenes) juran motivadas por un espíritu de independencia o a veces simplemente por eludir un matrimonio pactado sin que esto suponga el hacer caer en desgracia a toda la familia. Su juramento, realizado normalmente ante los doce hombres más ancianos del pueblo, consiste en permanecer vírgenes para siempre a cambio del derecho a vivir como hombres y ser respetadas y temidas como tales. Esto significa que nunca podrán tener un compañero y su renuncia a tener hijos por tanto, adquieren los derechos del hombre socialmente pero no en los roles matrimonial y sexual. Se despojan de su aspecto exterior femenino para adoptar las rudas maneras de los hombres montañeses. De su conversación con ellas concluye que viven con gran dignidad su historia; no son amargadas aunque reconocen que su decisión implica muchos sacrificios como es el de vivir en soledad y la castidad impuesta. No obstante, encuentra también quién se ha arrepentido de aquella decisión de adolescente, pero ahora ya es demasiado tarde. En Tropoja reconoce a la Hana de su libro en una camionera de 50 años que sueña con viajar a América con su hermana, para vestirse de mujer. Sabemos que la tradición no permite que una virgen jurada renuncie a su juramento dado que el castigo es la muerte. Dudamos que dicho castigo hoy se lleve a cabo aunque sin duda una ex virgen jurada sería apartada de la sociedad. Hoy esta virgen jurada, con la que la autora sigue en contacto, está comenzando la metamorfosis para reapropiarse de su propia existencia femenina.

Tras la publicación de esta novela y la difusión del documental sobre las vírgenes juradas realizado por Dones el fenómeno se ha transformado en un boom mediático convirtiéndose la zona en lugar de peregrinaje para turistas y periodistas curiosos. Ante este tratamiento superficial que se ha hecho del tema la autora nos aclara que ha no querido contar la historia de una tradición exótica o primitiva:

il mio libro non vuole raccontare storie esotiche e primitive dei nostri montanari arretrati, come qualcuno potrebbe pensare, volevo semplicemente scrivere un libro sulla solitudine di queste donne, su quello che succede a loro mentre il corpo invecchia, marcisce, si rinsecchisce, senza mai darsi a nessuno e senza mai ricevere né una carezza né un abbraccio. Per cui vorrei che venisse considerato un libro sulla solitudine di queste donne, su quello che succede a loro mentre il corpo invecchia, marcisce, si rinsecchisce, senza mai darsi a nessuno senza mai ricevere 
né una carezza né un abbraccio. Elvira scrittrice transnazionale, (Rukaj, www. osservatoriobalcani.org, 13/11/08)

\section{REFERECNIAS BIBLIOGRÁFICAS}

Clementi A., Elvira Dones, Un ponte tra due mondi, 5/11/2008, www.swissinfo.ch ,.

Dones, E., Senza bagagli, Lecce, BESA, 1998.

----, Sole bruciato, Milano, Feltrinelli (I Narratori), 2001.

----, Bianco giorno offeso, Novara, Interlinea, 2004.

----, I mari ovunque, Novara, Interlinea, 2007.

----, Vergine giurata, Milano, Feltrinelli, 2007.

Lepori P., Sei domande a Elvira Dones, http://www.culturactif.ch

Rukaj M., http://www.balcanicaucaso.org , 13/11/ 2008 